\title{
Testing (beliefs about) social preferences: Evidence from an experimental coordination game
}

\author{
Thorsten Chmura ${ }^{\mathrm{a}}$, Sebastian Kube ${ }^{\mathrm{b}, *}$, Thomas Pitz ${ }^{\mathrm{a}}$, Clemens Puppe \\ ${ }^{\mathrm{a}}$ University of Bonn, Germany \\ ${ }^{\mathrm{b}}$ University of Karlsruhe, Department of Economics and Business Engineering, Kollegium IV am Schloß, \\ 76128 Karlsruhe, Germany
}

Received 29 July 2004; received in revised form 25 January 2005; accepted 16 February 2005

Available online 23 May 2005

\begin{abstract}
We report experimental results on a simple coordination game in which two players can coordinate either on an equal distribution of payoffs or on a Pareto superior but unequal distribution of payoffs. We find that the higher the difference in individual payoffs, the less likely is a successful coordination on the Pareto superior distribution. While this is well in line with the recent models of inequity aversion, our results are best explained not by a preference for equality per se but rather by the belief that the opponent has such a preference.

(C) 2005 Elsevier B.V. All rights reserved.
\end{abstract}

Keywords: Experimental games; Fairness; Coordination games; Equilibrium selection

JEL classification: C72; C91; D63

\section{Introduction}

By now there seems to be broad agreement that in many contexts the traditional model of narrowly self-interested individuals is not the most useful description of economic agents. There is overwhelming experimental evidence that even in simple situations individual behavior involves more than just the maximization of one's own material payoff. In response to this evidence, a focus of recent research in

\footnotetext{
* Corresponding author. Tel.: +49 7216083081.

E-mail address: kube@wior.uni-karlsruhe.de (S. Kube).
} 
behavioral economics has been the question of how to model the "social preferences" of agents, i.e., the preferences over distributions of payoffs. Two influential approaches are the "inequity aversion models" of Bolton and Ockenfels (2000) and Fehr and Schmidt (1999) on the one hand, and the "quasi-maximin" model of Charness and Rabin (2002) on the other hand. The inequity aversion models presume that ceteris paribus agents prefer more equal distributions of payoffs, while the quasi-maximin model emphasizes both the role of the worst-off individual and of the aggregate payoff for the group. There are several studies that test the two approaches against each other. Engelmann and Strobel (2004), for instance, find that in their simple "dictator" experiments the influence of both efficiency concerns and maximin preferences is stronger than that of inequ(al)ity aversion; similar evidence is reported in Kritikos and Bolle (2001). By contrast, Güth et al. (2003) and Fehr et al. (2004) find that fairness concerns dominate efficiency concerns. Herreiner and Puppe (2004) study the relevance of efficiency versus equity considerations in a free-form bargaining context.

The purpose of the present study is to shed further light on the relative importance of fairness concerns versus efficiency concerns. Specifically, in our experimental design (to be described in the next section) "fairness concerns" are represented by an aversion to payoff differences between two players, while "efficiency concerns" correspond to a preference for (possibly unequal) distributions with higher total payoff; in most cases considered here, higher total payoff in fact means Pareto improvement, i.e., both players' payoff increases. Two main conclusions can be drawn from our results. First, the coordination on Pareto superior allocations is the more difficult the greater the asymmetry between the two players, i.e., the more unequal the resulting payoff distributions. In light of the evidence reported in Charness and Rabin (2002) and Engelmann and Strobel (2004), this re-establishes and confirms the importance of inequity aversion as modeled by Fehr and Schmidt (1999) and Bolton and Ockenfels (2000). Secondly, and perhaps even more importantly, our results suggest that it is not so much inequity aversion per se but rather the belief that others are driven by fairness concerns that best explains our observed behavior.

\section{Experimental setting and design}

The game underlying our experiments is the following two-person normal form game. Both players simultaneously choose either strategy $E$ or $F$. The resulting payoffs are common knowledge (Fig.1).

We conducted two experiments. The first consisted of seven treatments ([T1]-[T7]) with different values for the payoff vector $\left(x_{1}, x_{2}\right)$ resulting from the choice of $(E, E)$. Specifically, the treatments

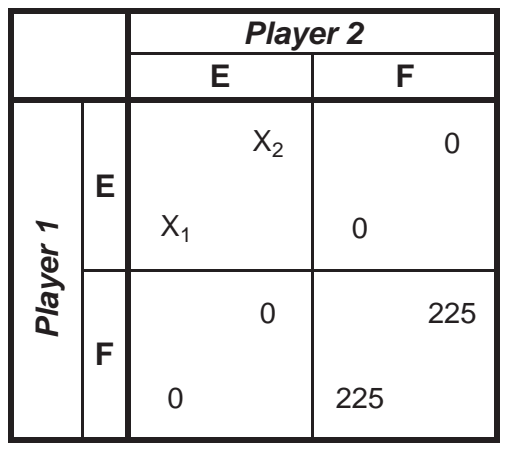

Fig. 1. Payoff matrix. 
Table 1

Relative frequency of decisions (player 1)

\begin{tabular}{lccccccc}
\hline Player 1 & {$[\mathrm{~T} 1]$} & {$[\mathrm{T} 2]$} & {$[\mathrm{T} 3]$} & {$[\mathrm{T} 4]$} & {$[\mathrm{T} 5]$} & {$[\mathrm{T} 6]$} & {$[\mathrm{T} 7]$} \\
\hline$x_{1}$ & 375 & 375 & 250 & 325 & 375 & 400 & 475 \\
$x_{2}$ & 200 & 225 & 250 & 250 & 250 & 250 & 250 \\
$E$ & $40 \%$ & $55 \%$ & $70 \%$ & $80 \%$ & $50 \%$ & $60 \%$ & $65 \%$ \\
$F$ & $60 \%$ & $45 \%$ & $30 \%$ & $20 \%$ & $50 \%$ & $40 \%$ & $35 \%$ \\
\hline
\end{tabular}

involved the following values: $\left(x_{1}, x_{2}\right)=(375,200)$ [T1], $(375,225)$ [T2], $(250,250)$ [T3], $(325,250)$ [T4], $(375,250)$ [T5] $(400,250)$ [T6] and $(475,250)$ [T7]. The second experiment featured the same game, but there we held $x_{2}$ fixed at 250 and let $x_{1}$ steadily increase from 175 to 475 in steps of 5 units, resulting in 61 different distributions.

If both players are purely selfish (and if this is common knowledge), the game represents a simple coordination game with two pure strategy Nash equilibria, namely $(F, F)$ (both choosing the "fair" outcome, i.e., the equal distribution $(225,225))$ and $(E, E)$ (both choosing the "efficient" outcome, i.e., the distribution $\left(x_{1}, x_{2}\right)$ which in almost all cases ${ }^{1}$ maximizes the sum of the payoffs). Note that in [T2]-[T7] the $(E, E)$ equilibrium is in fact Pareto superior to the $(F, F)$ equilibrium; the same holds in the second experiment for all $x_{1}>225$.

Our experiments were computerized ${ }^{2}$ and were conducted at the University of Bonn. Participants were recruited from the campus mensa and had no previous training in economics or game theory. Each participant played only one game and had to make exactly one decision. In total, 402 persons participated. In the first experiment, each treatment consisted of 20 games with 2 players, thus a total number of $7 \times 20 \times 2=280$ subjects participated in the first experiment. In the second experiment, each of the games corresponding to the different payoff distributions was played only once, hence $61 \times 2=122$ subjects participated in the second experiment. Each participant was informed about the game and his/her role as player 1 or player 2 , but not about who the other player was. The game was given in a matrix form, strategies were labeled $A$ and $B$, and the instructions ${ }^{3}$ were given in a neutral language to avoid framing effects.

After making their decision, subjects had also to fill out a questionnaire. Each subject received a lump sum payment of $1 €$ plus the individual payoff converted at a rate of 1 point $=0.01 €{ }^{4}$ If people failed to coordinate, they nevertheless got $1 €$. The average earning in the first experiment was $2.49 €$, with its minimum in [T3] at $1.88 €$ and its maximum in [T6] at $2.80 €$. The average earning in the second experiment was $2.94 €$. There were statistically significant differences in payoffs between the treatment pairs [T1][T5], [T1][T6], [T2][T5], [T2][T6], [T3][T4], [T3][T5], [T3][T6] and [T3][T7].

\section{Experimental results}

The experimental results from our first experiment are summarized in Tables 1-3. In the following, special attention will be given to treatments [T3]-[T7] because in each of these the distribution resulting

\footnotetext{
1 This is not true in the second experiment for distributions with $x_{1}<200$.

2 The program was written in PASCAL using RATImage by Abbink and Sadrieh (1995).

3 Instructions and screenshots are available from the authors upon request.

4 Thus, for instance, a payoff of 275 points corresponds to $2.75+1.00=3.75 € €$.
} 
Table 2

Relative frequency of decisions (player 2)

\begin{tabular}{lccccccc}
\hline Player 2 & {$[\mathrm{T} 1]$} & {$[\mathrm{T} 2]$} & {$[\mathrm{T} 3]$} & {$[\mathrm{T} 4]$} & {$[\mathrm{T} 5]$} & {$[\mathrm{T} 6]$} & {$[\mathrm{T} 7]$} \\
\hline$x_{1}$ & 375 & 375 & 250 & 325 & 375 & 400 & 475 \\
$x_{2}$ & 200 & 225 & 250 & 250 & 250 & 250 & 250 \\
$E$ & $40 \%$ & $70 \%$ & $65 \%$ & $65 \%$ & $70 \%$ & $70 \%$ & $75 \%$ \\
$F$ & $60 \%$ & $30 \%$ & $35 \%$ & $35 \%$ & $30 \%$ & $30 \%$ & $25 \%$ \\
\hline
\end{tabular}

from $(E, E)$ ("efficient" outcome) is a strict Pareto improvement relative to the "fair" outcome $(225,225)$. Player 2 always gets a payoff of 250. Player 1's payoff increases from 250 in [T3] to 475 in [T7], so we have an increasing inequality in payoffs between players 1 and 2 .

Under inequity aversion this has the following behavioral implications. Specifically, assume as in Fehr and Schmidt (1999) (henceforth: $[\mathbf{F} / \mathbf{S}]$ ), that player $i$ 's utility function $u_{i}$ is given by $u_{i}\left(x_{i}\right.$, $\left.x_{j}\right)=x_{i}-\alpha_{i} \max \left(x_{j}-x_{i}, 0\right)-\beta_{i} \max \left(x_{i}-x_{j}, 0\right)$, where $x_{i}$ and $x_{j}$ are the payoffs of player $i$ resp. $j$, and $\alpha_{i}$ and $\beta_{i}$ are parameters that measure $i$ 's degree of aversion against disadvantageous resp. advantageous inequality. As in [F/S], we assume that $\alpha_{i} \geq \beta_{i}$ and $0 \leq \beta_{i} \leq 1$. Simple calculations show that in [T3]-[T7] player 1 's best response is $E$ to $E$ and $F$ to $F$, and that he/she always prefers the efficient distribution to the fair distribution. By contrast, whether player 2 prefers the efficient distribution depends on her/his individual $\alpha$ and the specific payoff difference in $(E, E)$. The larger the difference, the less likely it is that a player 2 will prefer $(E, E)$ over $(F, F)$ in [T4]-[T7]. If $\alpha>250 /\left(x_{1}-250\right)$, strategy $E$ is strictly dominated by $F$ for player 2 , leaving the game with only one pure strategy Nash equilibrium, namely $(F, F) .^{5}$ Similar considerations apply to [T1] and [T2].

Tables 1 and 2 show the relative frequency of the observed strategy choices.

In [T3], $70 \%$ of player 1 choose $E$, in [T4] it is even $80 \%$. But then it drops significantly ${ }^{6}$ down to $50 \%$ in [T5], and although it rises again, it does not get beyond $65 \%$ in [T7]. On average, $65 \%$ of player 1 choose strategy $E$ in [T3]-[T7]. In light of the [F/S]-predictions, this is a relatively small percentage.

Looking at the behavior of player 2, we see a slightly different trend. In [T3] it starts with only $65 \%$ of subjects playing $E$, but then it rises steadily to $75 \%$ in [T7]. On average, $69 \%$ of player 2 choose strategy $E$ in [T3]-[T7]. Even if there are no significant differences between these treatments ${ }^{7}$, the number of player 2 choosing $E$ nonetheless rises with increasing inequity in $(E, E)$. This is surprising in view of the $[\mathbf{F} / \mathbf{S}]$ model, which would predict a declining number of choices of $E$ by player 2 . Note that even in the presence of inequity aversion, $(E, E)$ can still be a Nash equilibrium of our simple coordination game, as long as player 2's $\alpha$ does not get too large. Thus, the $[\mathbf{F} / \mathbf{S}]$ model certainly does not always rule out choices of strategy $E$ by either player. However, inequality aversion implies that the probability of the choice of $E$ by player 2 should decrease with increasing payoff difference, leaving the game with just one pure strategy Nash equilibrium $(F, F)$ in the extreme case.

Table 3 lists the observed distributions in the first experiment. The first row shows the number of games resulting in the efficient distribution, the second row the number of games resulting in the fair

\footnotetext{
5 Thanks to an anonymous referee for pointing this out. Note, however, that for a situation with just one equilibrium to arise, one needs a large $\alpha$. In [T4]-[T7], the required $\alpha$ value would imply a rejection of a $25000 /\left(x_{1}+250\right)$ share in an ultimatum bargaining game when the outside option equals 0 . For example, the $\alpha$ needed in [T7] would lead to a rejection of an offer of

6 Significant at 5\% level using a Fisher-Test. Other significant differences in behavior of player 1 are between [T1][T3] (5\% level), [T1][T4] (1\% level), [T1][T7] (10\% level) and [T2][T4] (10\% level).

${ }^{7}$ Significance (Fisher) of 5\% in [T1][T2], [T1][T5], [T1][T6], [T1][T7], and of 10\% in [T1][T3], [T1][T4].
} 
Table 3

Resulting distributions

\begin{tabular}{llllllllll}
\hline & {$[\mathrm{T} 1]$} & {$[\mathrm{T} 2]$} & {$[\mathrm{T} 3]$} & {$[\mathrm{T} 4]$} & {$[\mathrm{T} 5]$} & {$[\mathrm{T} 6]$} & {$[\mathrm{T} 7]$} & {$[\mathrm{T} 3]-[\mathrm{T} 7]$} & {$[\mathrm{T} 1]-[\mathrm{T} 7]$} \\
\hline$(E, E)$ & 4 & 7 & 7 & 10 & 8 & 9 & 9 & 43 & 54 \\
$(F, F)$ & 8 & 2 & 0 & 1 & 4 & 3 & 1 & 9 & 19 \\
$(E, F)$ & 4 & 4 & 7 & 6 & 2 & 3 & 4 & 22 & 30 \\
$(F, E)$ & 4 & 7 & 6 & 3 & 6 & 5 & 6 & 26 & 37 \\
\hline
\end{tabular}

distribution. The third and fourth rows give the number of coordination failures. Obviously, more games result in the efficient distribution than in the fair distribution. This holds for all treatments except for [T1]. In [T3]-[T7], we observe 43 efficient endings and only 9 fair ones. This might give the impression that people are driven mainly by efficiency concerns, but it is in fact not evident. If we look at the number of games in which one player chooses $E$ while the other chooses $F$, we see that nearly half of all games fall into this category (67 out of 140). Thus, efficiency concerns can at least not be common knowledge.

Taking a closer look, one can distinguish two different cases of coordination failure. Either player 1 or player 2 can be made "responsible" for not reaching the efficient distribution by choosing strategy $F$. Remarkably, the pattern of coordination failures changes from [T3] to [T7]. In [T3] we observe 6 instances of $(F, E)$ versus 7 instances of $(E, F)$, and in [T4] 3 instances of $(F, E)$ versus 6 instances of $(E, F)$, thus in these two treatments the failure to reach the efficient outcome is more often due to player 2's choice. By contrast, as is evident from Table 3, in [T5]-[T7] it is more often player 1 who is responsible for not reaching the efficient outcome. Note that in the $[\mathbf{F} / \mathbf{S}]$ model one would expect the opposite pattern of behavior.

Inequity aversion does seem to influence players' choices, but in a complex way. A clue can be found by analyzing the questionnaires. When asked for the reason of their decision, many subjects wrote that they tried to anticipate the other player's choices and determined their own strategy based on that belief. In our simple coordination game, we may thus take the actual strategy choice as an estimator of a player's belief. In view of this, our results suggest that it is not so much inequity aversion per se that matters but rather the belief that the other player is inequity averse. ${ }^{8}$ Not surprisingly, the assessment of the situation seems to be sensitive to the magnitude of the payoff difference in the efficient distribution. In [T3] and [T4], when the difference is small, many player 1 think that player 2 will choose $E$ because they both can earn more by doing so, so we see a high percentage of player 1 choosing $E$. Around [T5], there seems to be a turning point. Player 1 now seems to think that the other player regards the efficient distribution as unfair, so we see many player 1 choosing $F$. In the extreme, if player 1 believes that player 2's inequity aversion is large enough to prefer $(0,0)$ over $\left(x_{1}, x_{2}\right)$, then in equilibrium player 1 must choose $F$ because for player $2 F$ is a dominant strategy now. By contrast, player 2 seems to think that player 1 will choose $E$ because his/her payoff increases significantly, so in order to coordinate player 2 chooses $E$. Thus, people appear to think too "badly" about the other player's attitude, and the strength of this effect seems to be influenced by the size of the payoff difference resulting from $(E, E)$.

To further examine this, we conducted the second experiment already described above. The results are summarized by Figs. 2 and 3.

\footnotetext{
${ }^{8}$ Unfortunately, the players are often wrong in their estimation of the other player's behavior, so they frequently fail to coordinate.
} 


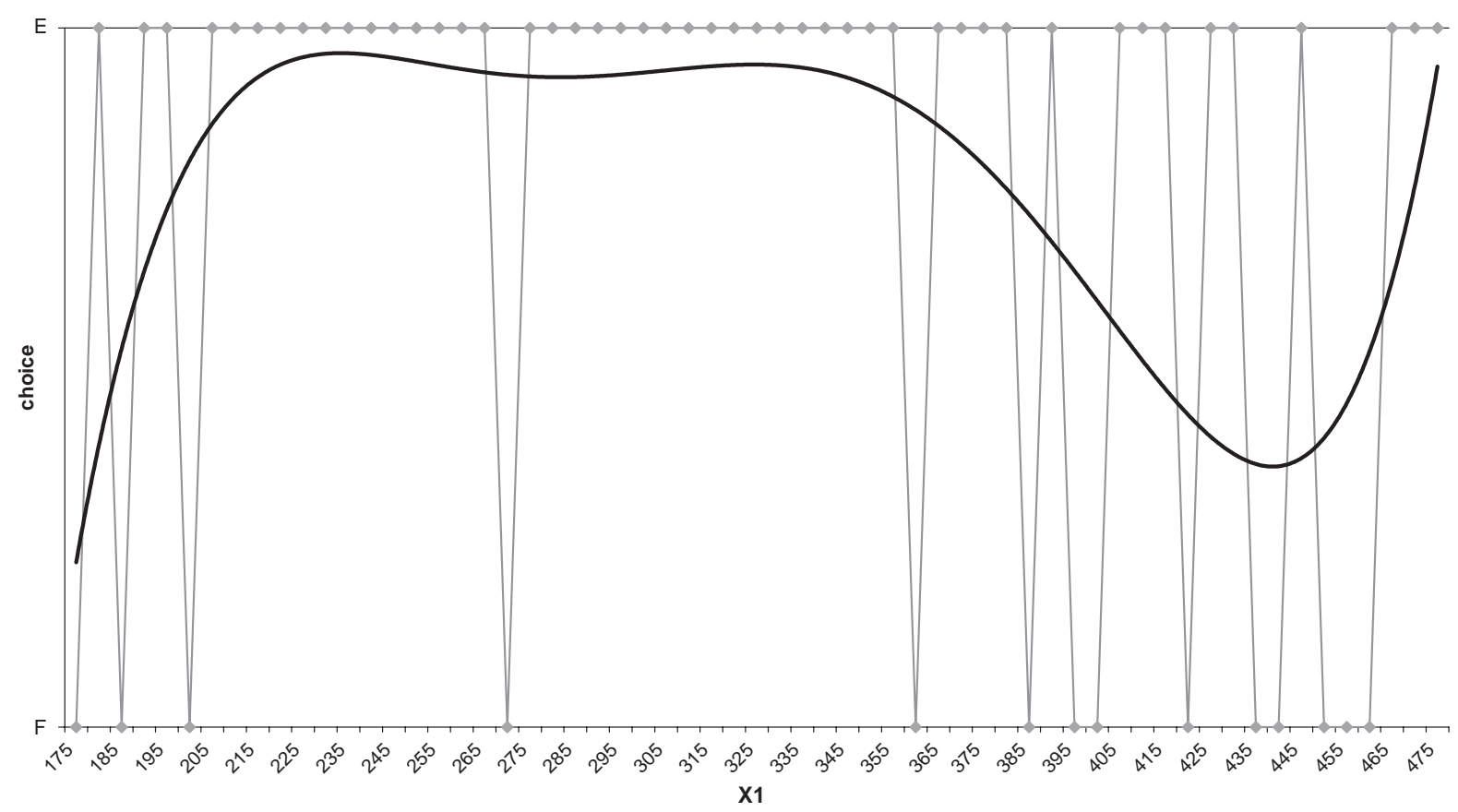

Fig. 2. Choice player 1.

Fig. 2 shows the choices of player 1 for each $x_{1}\left(175 \leq x_{1} \leq 475\right)$, Fig. 3 shows the corresponding choices of player 2. A dot at the top marks a choice of strategy $E$, a dot at the bottom a choice of $F$. ${ }^{9}$

Qualitatively, the trend for player 1 decisions is similar to the behavior observed in the first experiment. For low $x_{1}$ values they mostly choose $E$. Then, around $x_{1}=360$, they seem to start to think that the other player may choose $F$ and play $F$ more often. ${ }^{10}$ For high $x_{1}$ values, fairness concerns are no longer dominant and the choice of $E$ is observed more often. The behavior of player 2 corresponds with the results from our first experiment as well. After a variable beginning there is a remarkably long period (from $x_{1}=325$ to $x_{1}=440$ ) with the constant choice of $E$. At the end the inequality seems to get too large and $F$ is again chosen sometimes. For both players, some choices of $F$ are observed at high $x_{1}$ values after a period of constant choice of $E$. Remarkably, this period ends much later for player 2 than for player 1, which again confirms the conjecture that the qualitative nature of our observations is due to player 1's beliefs rather than player 2's actual social preferences.

Combining the choices, we see that in $62 \%$ of the cases the games result in the efficient distribution. Only in $7 \%$ of the cases the fair outcome results, and in $31 \%$ of all cases the players fail to coordinate and earn zero payoff. We thus have a much lower number of coordination failures than in our first experiment, and a much higher number of efficient endings. ${ }^{11}$

\footnotetext{
${ }^{9}$ The curves represent a polynomial trend (of fifth degree); they only serve for visualization of the results.

${ }^{10}$ One could think that they care for fairness, but in the questionnaires many individuals explicitly wrote that they chose $F$ because they thought that the other player may do so.

${ }^{11}$ However, each $x_{1}$ value was played only once in our second experiment, so the data basis is much weaker than in the first experiment.
} 


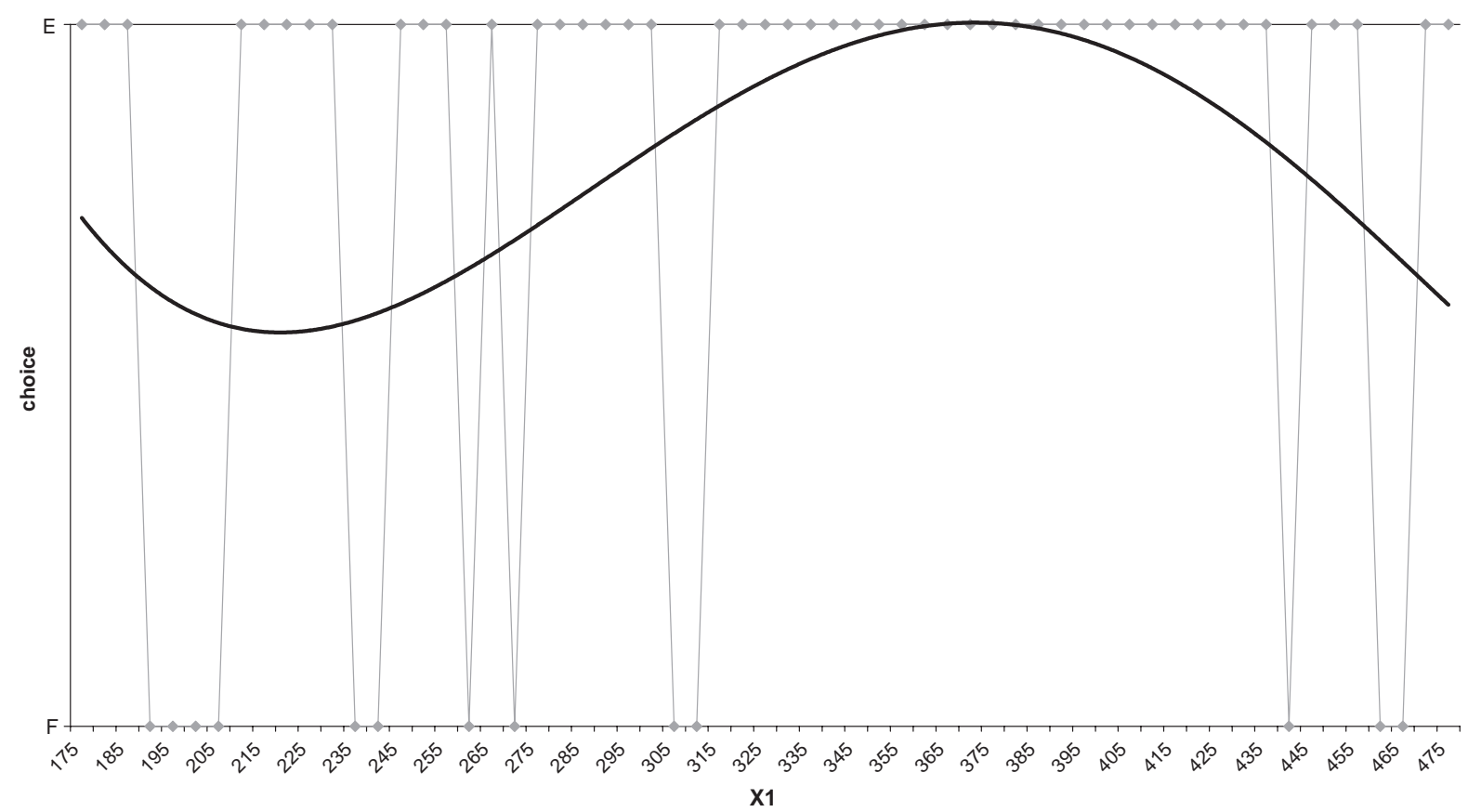

Fig. 3. Choice player 2.

Summarizing, we find that efficiency concerns are important. But if inequality gets significant, difference aversion hampers the coordination on an efficient and even Pareto superior outcome. Thus, the disregard of equality in favor for unanimous improvement is at least not common knowledge.

\section{References}

Abbink, K., Sadrieh, K., 1995. RATImage-Research Assistance Toolbox for Computer-Aided Human Behavior Experiments. SFB Discussion Paper B-325, University of Bonn.

Bolton, G., Ockenfels, A., 2000. ERC: a theory of equity, reciprocity and competition. American Economic Review 90, $269-299$.

Charness, G., Rabin, M., 2002. Understanding social preferences with simple tests. Quarterly Journal of Economics 117, $817-869$.

Engelmann, D., Strobel, M., 2004. Inequality aversion, efficiency and maximin preferences in simple distribution experiments. American Economic Review 94 (4), 857-869.

Fehr, E., Schmidt, K., 1999. A theory of fairness, competition and cooperation. Quarterly Journal of Economics 114, 817-868. Fehr, E., Naef, M., Schmidt, K., 2004. The role of equality and efficiency in social preferences. Mimeographed.

Güth, W., Kliemt, H., Ockenfels, A., 2003. Fairness versus efficiency: an experimental study of (mutual) gift giving. Journal of Economic Behavior and Organization 50, 465-475.

Herreiner, D., Puppe, C., 2004. Equitable Allocations in Experimental Bargaining Games: Inequality Aversion versus Efficiency. Mimeographed.

Kritikos, A., Bolle, F., 2001. Distributional concerns equity- or efficiency-oriented? Economics Letters 73, 333-338. 